\title{
BMJ Open Consultations in general practices with and without mental health nurses: an observational study from 2010 to 2014
}

\author{
Tessa Magnée, ${ }^{1}$ Derek P de Beurs, ${ }^{1}$ Dinny H de Bakker, ${ }^{1,2}$ Peter F Verhaak ${ }^{1,3}$
}

To cite: Magnée T, de Beurs DP, de Bakker DH, et al. Consultations in general practices with and without mental health nurses: an observational study from 2010 to 2014. BMJ Open 2016;6:e011579. doi:10.1136/bmjopen-2016011579

- Prepublication history and additional material is available. To view please visit the journal (http://dx.doi.org/ 10.1136/bmjopen-2016011579).

Received 18 February 2016 Revised 7 June 2016 Accepted 9 June 2016

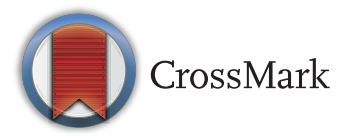

\footnotetext{
${ }^{1}$ Netherlands Institute of Health Services Research (NIVEL), Utrecht, The Netherlands ${ }^{2}$ Tilburg University, Scientific Centre for Transformation in Care and Welfare (TRANZO), Tilburg, The Netherlands ${ }^{3}$ Department of General Practice, Groningen University, Groningen, The Netherlands
}

Correspondence to Tessa Magnée; t.magnee@nivel.nl

\section{ABSTRACT}

Objectives: To investigate care for patients with psychological or social problems provided by mental health nurses (MHNs), and by general practitioners (GPs) with and without MHNs.

Design: An observational study with consultations recorded by GPs and MHNs.

Setting: Data were routinely recorded in 161-338 Dutch general practices between 2010 and 2014 .

Participants: All patients registered at participating general practices were included: 624477 patients in 2010 to 1392187 patients in 2014.

Outcome measures: We used logistic and Poisson multilevel regression models to test whether GPs recorded more patients with at least one consultation for psychological or social problems and to analyse the number of consultations over a 5-year time period. We examined the additional effect of an MHN in a practice, and tested which patient characteristics predicted transferral from GPs to MHNs.

Results: Increasing numbers of patients with psychological or social problems visit general practices. Increasing numbers of GPs collaborate with an MHN. GPs working in practices with an MHN record as many consultations per patient as GPs without an $\mathrm{MHN}$, but they record slightly more patients with psychological or social problems ( $\mathrm{OR}=1.05 ; 95 \%$ $\mathrm{Cl} 1.02$ to 1.08). MHNs most often treat adult female patients with common psychological symptoms such as depressive feelings.

Conclusions: MHNs do not seem to replace GP care, but mainly provide additional long consultations. Future research should study to what extent collaboration with an MHN prevents patients from needing specialised mental healthcare.

\section{INTRODUCTION}

Mental disorders are highly common in developed countries and account for a large burden of disease. ${ }^{1}$ The rising costs of mental healthcare are a major reason for concern for many governments. ${ }^{2}$ Therefore, the WHO underlines the importance of strengthening primary mental healthcare, where good quality services are highly accessible and

\section{Strengths and limitations of this study}

- This was the first study to quantitatively investigate a novel phenomenon in primary care: nurses with mental health expertise working in general practices.

- Data from 5 years of a very large sample of patients were analysed.

- The study is descriptive rather than causative, because of the observational design.

- We analysed the recording of mental health problems by general practitioners, not the actual prevalence of psychological and social problems.

- The (methods of) screening and assessment of psychological or social problems is likely to vary between practices.

relatively inexpensive. ${ }^{3}$ However, many general practitioners (GPs) do not have the resources, time and expertise to treat all patients with psychological or social problems themselves. ${ }^{2}$ As a result, increasing numbers of patients are treated in specialised care. ${ }^{45}$

In 2014, the Dutch government introduced a reform of the Dutch mental healthcare system, to stimulate a shift of patients from specialised to primary care. Since then, GPs can only refer patients with psychiatric disorders according to DSM-IV criteria (Diagnostic and Statistical Manual of Mental Disorders, Fourth Edition ${ }^{6}$ ) to other professionals working in mental healthcare. All patients without a psychiatric disorder should be treated within general practice.

A promising method to prepare general practice for this intensified role in the mental healthcare system is the shifting of care from GPs to nurses. Increasing numbers of GPs work together with a nurse with expertise in mental health: a mental health nurse (MHN). Since 2014, a GP working in an average size practice can be supported by an MHN for $\sim 1$ day a week. Previous research on shifting care from doctors to nurses has focused mainly on patients with somatic diseases. Shifting care to nurses may reduce the 
increasing workload of GPs, ${ }^{7}$ improve the accessibility of $\mathrm{care}^{8}$ and reduce the number of patients who need referral to specialised care. Patients are satisfied when they receive care from nurses instead of from doctors. ${ }^{9}$ Nurses working in general practice are probably more costeffective than GPs. ${ }^{7} 10^{11}$ Besides, nurses in general practice can treat certain patients who otherwise would have been treated in more expensive specialised care. ${ }^{12}$

Dutch MHNs received higher vocational training in nursing or psychology, and their main tasks are to perform diagnostic research, to improve the quality of the referral to other mental health caregivers and to deliver short-term care (such as counselling) to patients with psychological symptoms or social problems. MHNs work under the supervision of the GP. In general, the GP decides after a first consultation if a patient should visit the MHN. GPs can also decide to treat patients themselves, or refer patients to specialised mental healthcare. Treatment of (mild) psychological or social problems provided in primary care seems effective, more accessible than treatment in specialised care and leads to satisfaction among patients and caregivers. ${ }^{13-16}$

It is not clear to what extent GP care is replaced by MHN care. It is plausible that GPs collaborating with an MHN see a smaller number of patients with psychological or social problems themselves, or use fewer or shorter consultations per patient. On the other hand, is it possible that nurses generate an extra demand for care $^{7}$ or uncover needs previously unmet by the GP, and deliver additional consultations to patients with psychological or social problems?

The objective of this study was to examine a possible shift of care from GPs to MHNs between 2010 and 2014. Using a primary care database, we investigated (1) how many patients with psychological problems or social problems were recorded by GPs and MHNs yearly, (2) how many consultations (total and long) were recorded per patient by GPs and MHNs, (3) if the total number of patients and consultations changed over the years, (4) if differences between GPs working with an MHN and GPs without an MHN existed and (5) which patients were most often treated by the MHN after they visited the GP.

\section{METHODS}

\section{Database}

In an observational study, anonymised data from 2010 to 2014 from electronic health records of general practices participating in the NIVEL Primary Care Database (NIVEL-PCD) ${ }^{17}$ were analysed. All caregivers participating in NIVEL-PCD routinely record care they deliver to their patients. NIVEL-PCD has a dynamic nature; the number of participating general practices varies over time and thus varied over our study period. In general, the number of participating practices increases every year, but practices can also discontinue participation. The general practices $(n=161$ in 2010 to $n=338$ in 2014; see online supplementary file) and their patient populations are representative of Dutch general practices and the Dutch population, although group practices and practices in non-urban areas are somewhat over-represented compared with national numbers. ${ }^{18}$ Only practices which met a quality criterion for recording ( $\geq 70 \%$ complete) were included in this study.

\section{Data}

In the Netherlands, all (17 million) citizens are registered at a general practice. All patients registered at participating practices were included in the study ( $\mathrm{n}=624477$ in 2010 to $\mathrm{n}=1392187$ in 2014; see online supplementary file for age and gender of patients). Dutch GPs receive a capitation fee for each registered patient, and receive additional fees for recorded consultations. Dutch GPs and MHNs use their own standardised codes to record standard and long (over $20 \mathrm{~min}$ ) consultations with patients. They record at least one and up to three diagnoses according to the International Classification of Primary Care (ICPC) system per consultation, based on their clinical evaluation. Only consultations with at least one diagnosis concerning psychological or social problems were selected for this study, including all psychological problems (psychological symptoms or disorders) and social problems (table 1).

The recorded consultations were office consultations (standard or long), home consultations (standard or long), and telephone and email consultations (standard only). These consultations cover the majority of GP work regarding direct care to patients. When a patient had multiple recorded psychological or social diagnoses at one consultation, the first diagnosis over-ruled the second or third diagnosis, and the second over-ruled the third. When a patient attended multiple consultations concerning psychological or social problems in a year, the diagnosis of the last (GP or MHN) consultation was regarded as the main diagnosis of the patient. This was assumed to be the most adequate method, as psychological diagnostics usually take place over multiple consultations. Most patients visited the GP for only one type of problems during a year. Gender and age were recorded for all patients.

Table 1 Psychological or social problems included in the study, coded with the International Classification of Primary Care (ICPC) system

Type of psychological or social problems ICPC code

Psychological symptoms

Psychological disorders P01-P29, for example, P01-anxious feelings P70-P99, for example, P74-anxiety disorder Social problems 
Data on practice level were available from the NIVEL-PCD database administration. Degree of urbanisation was categorised as very high $(>2500$ addresses per $\left.\mathrm{km}^{2}\right)$, high (1500-2500 addresses per $\mathrm{km}^{2}$ ), medium (1000-1500 addresses per $\left.\mathrm{km}^{2}\right)$, low $(500-1000$ addresses per $\left.\mathrm{km}^{2}\right)$ or very low $(<500$ addresses per $\mathrm{km}^{2}$ ). Practice type was defined as solo (one GP), duo (two GPs) or group practice (GP collaborating with at least two other GPs or with other primary care professionals). Practice size was categorised as small $(<2500$ enlisted patients), medium (2500-3499 patients) or large ( $\geq 3500$ patients). Practices with at least 25 recorded consultations by MHNs use in a given year were categorised as practices with an MHN. Practices with an MHN were more often group practices, located in urban areas, and had a somewhat younger patient population (see online supplementary file); we corrected analyses for these variables.

\section{Analyses}

We provide basic descriptive statistics. We report how many GPs worked with an MHN per year. Next, we present the average number of consultations recorded by GPs and MHNs for psychological or social problems.

We analysed three different dependent variables: (1) attending at least one consultation for psychological or social problems in a given year per patient (no or yes), (2) the total number of consultations for psychological or social problems per year per patient and (3) the number of long consultations for psychological or social problems per year per patient. Six analyses were performed in total, as the three outcome measures were analysed separately for GPs and MHNs.

Associations between consultations for psychological or social problems and patient and practice characteristics were analysed with multilevel generalised repeated measurements regression analyses using a model with three hierarchical levels (measurement, patient and general practice) to account for clustered data. At the patient level, a full, unstructured variance/covariance matrix for the years was fitted to control for the autocorrelation between years within patients. At the practice level, only a yearly between practice variance was fitted. Adding covariances made the models unstable, because only a limited number of practices provided data for several years. The intraclass correlation coefficient (ICC) at the practice level was calculated for each year to estimate variation between practices. ${ }^{19}$ Although the ICC is difficult to interpret in logistic models, it does allow us to compare the level of between practice variation over the years. Binary outcomes were analysed using logistic regression and count outcomes with Poisson regression.

While analysing recorded GP consultations, we were interested in the effect of year and the effect of the presence of an MHN at a practice. Regarding MHN consultations, we were interested in the effect of year and patient characteristics (ICPC diagnosis, age and gender of patients). We wanted to compare patients exclusively visiting the GP with patients also visiting the MHN. Therefore, while analysing MHN consultations, we only included patients who visited the GP at least once in a given year for psychological or social problems. Thereby, we could compare GP patients with MHN patients. All analyses were corrected for degree of urbanisation of practice location, practice type, practice size, gender and age of patients, and the part of the year that patients were registered at a practice. The significance level was set at $<0.05$. All analyses were performed using MLwiN V.2.30.

\section{RESULTS}

\section{Patients treated by GPs and MHNs}

Between 2010 and 2014, the percentage of general practices with an MHN increased from 20\% to $83 \%$ (see online supplementary file). Figure 1 shows that in 2014, both GPs (table 2A) and MHNs (table 3A) treated an increased number of patients with psychological or social problems compared with 2010. GPs in practice with an MHN treated slightly more patients with psychological or social problems than GPs without an MHN ( $\mathrm{OR}=1.05 ; 95 \%$ CI 1.02 to 1.08 ). The intraclass correlation at the practice level ranged from $1.5 \%$ in 2010 to $1.2 \%$ in 2014 .

\section{Number of consultations}

Yearly, patients with psychological or social problems had more than two GP consultations and relatively few long GP consultations (figure 2). Patients who visited the MHN had around three consultations per year and especially long consultations. Between 2010 and 2014, the number of consultations per patient increased for both GPs (table 2B) and MHNs (table 3B). The number of long GP consultations (table 2C) and long MHN consultations (table 3C) increased as well. Overall, we found no differences in the number of total or long GP consultations used per patient in general practices with or without an MHN (table 2B,C).

\section{Patients visiting the MHN}

Some of the GP patients with psychological or social problems were more likely to have at least one MHN consultation (table 3A): patients with depressive feelings, neurasthenia, relational problems with a partner, stress, anxious feelings, relational problems with a child or depression, than patients with problems categorised as 'other psychological symptoms' (table 3A). Females were more likely to have at least one MHN consultation than males. Older patients, especially 19-44 years old, were more likely to have at least one MHN consultation than children.

Other GP patients were less likely to have at least one MHN consultation in comparison to patients with other psychological symptoms: patients diagnosed with tobacco abuse, learning problems, dementia, sleeping 
Figure 1 Numbers of patients with at least one consultation for psychological or social problems at Dutch GPs and MHNs per 1000 registered patients between 2010 and 2014 (crude rates). GP, general practitioner; MHN, mental health nurse.

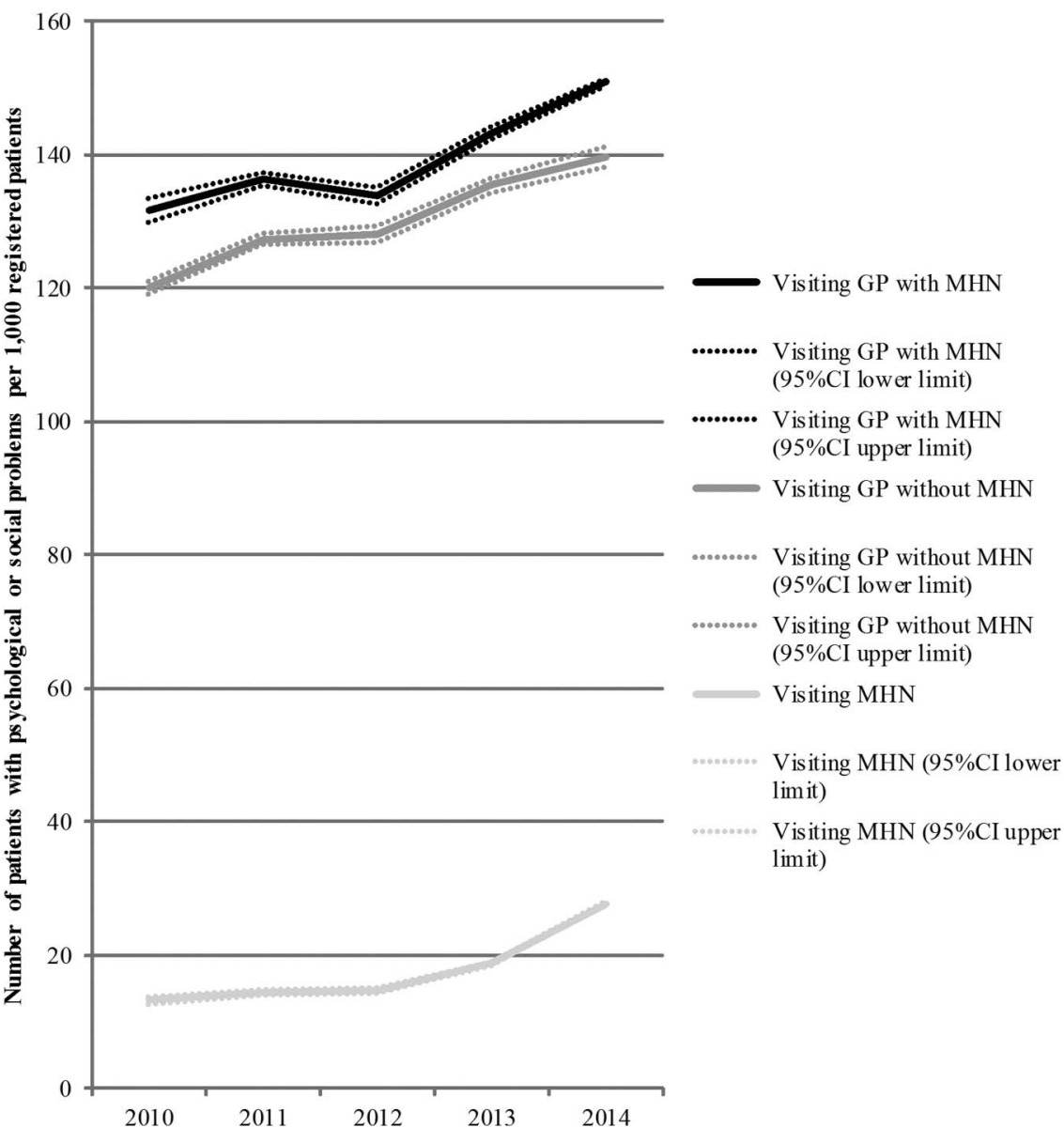

problems, concentration or memory problems, alcohol abuse, child hyperactivity, other psychological disorders, loss of a partner, an ill partner, other child behavioural problems or other social problems.

In general, patient characteristics that increased the chance of having at least one MHN consultation also increased the likeliness of having a higher number of consultations for patients with at least one MHN consultation. For example, females, adult patients and patients with depressive feelings or neurasthenia who visited the MHN were more likely to have a higher number of total (table 3B) or long MHN consultations (table 3C).

\section{DISCUSSION}

\section{Principal findings}

Between 2010 and 2014, the percentage of Dutch general practices with an MHN increased considerably from $20 \%$ to $83 \%$. MHNs as well as GPs record increasing numbers of patients with psychological or social problems, as well as an increasing number of consultations per patient. The availability of an MHN at a practice is associated with a slightly larger number of patients seen by the GP, but not with a lower number of GP consultations per patient. The chance on MHN treatment was highest for female adult patients with common psychological or social problems, such as depressive or anxious feelings, neurasthenia, stress or relational problems.

\section{Strengths and weaknesses}

Since this is a descriptive study, we cannot draw any conclusions on causality. A major strength of this study is that we were able to analyse data from 5 years for a very large sample of patients. Most practices that participated in NIVEL-PCD do so for several years. GPs only receive fees for their services when they record the care they provide, are trained in coding and record according to a most complete records were used for analyses. An important limitation of our study is the presumption that mental health problems are often under-reported in patient health records. When mental health problems appear together with chronic illness or vague somatic symptoms, as they often do, the GP is likely to record a somatic problem. Therefore, our results probably underestimate the absolute number of patients with psychological or social problems in general practice. Another important limitation is that GPs vary in screening skills and in assessment methods of mental health problems, resulting in between practice variation. By adding a random intercept at the practice level in our analysis, we controlled for any influence of practice variation. Also, national guideline. Only data from practices with the 
Table 2 Statistics for multivariate multilevel tests of the effects of time and practice and patient characteristics on consultations by Dutch GPs

\begin{tabular}{|c|c|c|c|c|c|c|}
\hline \multirow{3}{*}{$\begin{array}{l}\text { Number of } \\
\text { observations } \\
\text { in analyses }\end{array}$} & \multicolumn{2}{|c|}{$\begin{array}{l}\text { (A) Having at least one GP } \\
\text { consultation for } \\
\text { psychological or social } \\
\text { problems per patient per } \\
\text { year (no/yes) }\end{array}$} & \multirow{2}{*}{\multicolumn{2}{|c|}{$\begin{array}{l}\text { (B) Number of GP } \\
\text { consultations for } \\
\text { psychological or social } \\
\text { problems per patient per } \\
\text { year } \\
\mathrm{N}=594231 \text { (only patients } \\
\text { with at least one GP } \\
\text { consultation for } \\
\text { psychosocial problems) }\end{array}$}} & \multirow{2}{*}{\multicolumn{2}{|c|}{$\begin{array}{l}\text { (C) Number of long GP } \\
\text { consultations for } \\
\text { psychological or social } \\
\text { problems per patient per } \\
\text { year } \\
\mathrm{N}=594231 \text { (only patients } \\
\text { with at least one GP } \\
\text { consultation for } \\
\text { psychosocial problems) }\end{array}$}} \\
\hline & \multicolumn{2}{|c|}{$\begin{array}{l}\mathrm{N}=4389048 \text { (all GP } \\
\text { patients) }\end{array}$} & & & & \\
\hline & OR & $95 \% \mathrm{Cl}$ & $\mathbf{R R}$ & $95 \% \mathrm{Cl}$ & $\mathbf{R R}$ & $95 \% \mathrm{Cl}$ \\
\hline \multicolumn{7}{|c|}{ Year } \\
\hline \multicolumn{7}{|c|}{2010 (reference) } \\
\hline 2011 & 1.05 & 1.01 to 1.11 & 1.03 & 1.00 to 1.05 & 1.02 & 0.96 to 1.09 \\
\hline 2012 & 1.07 & 1.02 to 1.13 & 1.00 & 0.98 to 1.02 & 1.03 & 0.96 to 1.10 \\
\hline 2013 & 1.15 & 1.10 to 1.21 & 1.05 & 1.03 to 1.08 & 1.07 & 1.00 to 1.14 \\
\hline 2014 & 1.23 & 1.17 to 1.28 & 1.12 & 1.09 to 1.15 & 1.13 & 1.06 to 1.21 \\
\hline \multicolumn{7}{|c|}{ Practice characteristics } \\
\hline \multicolumn{7}{|c|}{ Employment of MHN } \\
\hline Yes & 1.05 & 1.02 to 1.08 & 1.00 & 0.98 to 1.02 & 1.01 & 0.97 to 1.06 \\
\hline \multicolumn{7}{|c|}{ Practice type } \\
\hline \multicolumn{7}{|c|}{ Solo (reference) } \\
\hline Duo & 1.05 & 1.01 to 1.09 & 1.02 & 1.00 to 1.05 & 1.09 & 1.02 to 1.16 \\
\hline \multicolumn{6}{|c|}{ Degree of urbanisation (\%) } & 0.98 to 1.10 \\
\hline \multicolumn{7}{|c|}{$\begin{array}{l}\text { Very high } \\
\text { (reference) }\end{array}$} \\
\hline High & 0.99 & 0.95 to 1.03 & 1.04 & 1.02 to 1.07 & 1.04 & 0.98 to 1.10 \\
\hline Medium & 0.90 & 0.86 to 0.93 & 1.04 & 1.01 to 1.06 & 1.13 & 1.06 to 1.21 \\
\hline Low & 0.77 & 0.74 to 0.80 & 1.05 & 1.02 to 1.08 & 1.20 & 1.12 to 1.28 \\
\hline Very low & 0.81 & 0.77 to 0.85 & 1.08 & 1.05 to 1.11 & 1.24 & 1.15 to 1.33 \\
\hline \multicolumn{7}{|c|}{ Practice size } \\
\hline \multicolumn{7}{|c|}{ Small (reference) } \\
\hline Medium & 1.01 & 0.97 to 1.04 & 1.03 & 1.00 to 1.05 & 1.00 & 0.94 to 1.06 \\
\hline Large & 1.03 & 0.99 to 1.07 & 1.03 & 1.01 to 1.06 & 1.06 & 0.99 to 1.13 \\
\hline \multicolumn{7}{|c|}{ Patient characteristics } \\
\hline \multicolumn{7}{|c|}{ Sex } \\
\hline \multicolumn{7}{|c|}{ Male (reference) } \\
\hline Female & 1.43 & 1.42 to 1.44 & 1.07 & 1.05 to 1.08 & 1.13 & 1.10 to 1.15 \\
\hline \multicolumn{7}{|c|}{ Age category (years) } \\
\hline \multicolumn{7}{|c|}{ 0-19 (reference) } \\
\hline $19-44$ & 1.68 & 1.67 to 1.70 & 1.33 & 1.31 to 1.36 & 1.96 & 1.88 to 2.04 \\
\hline $45-64$ & 1.94 & 1.92 to 1.96 & 1.41 & 1.38 to 1.44 & 2.13 & 2.04 to 2.22 \\
\hline$>65$ & 2.36 & 2.33 to 2.39 & 1.59 & 1.55 to 1.62 & 2.06 & 1.98 to 2.16 \\
\hline
\end{tabular}

since the ICC remained stable over the years (between $1.5 \%$ and $1.2 \%$ ), we argue that it is legitimate to compare the records over the years, as the bias due to differences in screening and assessment methods seems to be similar for each year. Unfortunately, referral records to specialised care were not complete and thus could not be used for this study.

Group practices were modestly over-represented in our study. Since group practices more often have resources to employ an MHN compared with solo GP practices, this could mean our data showed a small overestimation of practices with an MHN. On the other hand, practices in urban locations, which collaborate more often with an MHN, were under-represented. The percentage of practices with an MHN that we found was comparable to the number from a national GP survey. ${ }^{20}$

We used recorded consultations to determine if a practice had employed an MHN or not in a given year. 
Table 3 Statistics for multivariate multilevel tests of the effects of time and practice and patient characteristics on consultations by Dutch MHNs

\begin{tabular}{|c|c|c|c|c|c|c|}
\hline \multirow[b]{3}{*}{ Number of observations in analyses } & \multirow{2}{*}{\multicolumn{2}{|c|}{$\begin{array}{l}\text { (A) Having at least one MHN } \\
\text { consultation per patient per } \\
\text { year (no/yes) } \\
\mathrm{N}=372281 \text { (only patients with } \\
\text { psychological or social } \\
\text { problems) }\end{array}$}} & \multirow{2}{*}{\multicolumn{2}{|c|}{$\begin{array}{l}\text { (B) Number of MHN } \\
\text { consultations per patient per } \\
\text { year }\end{array}$}} & \multirow{2}{*}{\multicolumn{2}{|c|}{$\begin{array}{l}\text { (C) Number of long MHN } \\
\text { consultations per patient per } \\
\text { year }\end{array}$}} \\
\hline & & & & & & \\
\hline & OR & $95 \% \mathrm{Cl}$ & $\overline{\mathbf{R R}}$ & $95 \% \mathrm{Cl}$ & $\overline{\mathbf{R R}}$ & $95 \% \mathrm{Cl}$ \\
\hline \multicolumn{7}{|l|}{ Year } \\
\hline \multicolumn{7}{|l|}{2010 (reference) } \\
\hline 2011 & 1.18 & 0.97 to 1.44 & 1.27 & 1.05 to 1.53 & 1.24 & 1.03 to 1.51 \\
\hline 2012 & 1.16 & 0.95 to 1.41 & 1.21 & 1.00 to 1.47 & 1.20 & 0.99 to 1.47 \\
\hline 2013 & 1.44 & 1.20 to 1.73 & 1.62 & 1.35 to 1.94 & 1.56 & 1.30 to 1.88 \\
\hline 2014 & 2.03 & 1.70 to 2.43 & 2.23 & 1.87 to 2.65 & 2.15 & 1.80 to 2.56 \\
\hline \multicolumn{7}{|l|}{ Practice characteristics } \\
\hline \multicolumn{7}{|l|}{ Practice type } \\
\hline \multicolumn{7}{|l|}{ Solo (reference) } \\
\hline Duo & 1.02 & 0.89 to 1.17 & 0.98 & 0.86 to 1.12 & 0.99 & 0.87 to 1.13 \\
\hline Group & 0.96 & 0.86 to 1.08 & 1.01 & 0.90 to 1.13 & 0.99 & 0.88 to 1.11 \\
\hline \multicolumn{7}{|l|}{ Degree of urbanisation (\%) } \\
\hline \multicolumn{7}{|l|}{ Very high (reference) } \\
\hline High & 1.14 & 1.01 to 1.28 & 1.12 & 1.00 to 1.26 & 1.14 & 1.02 to 1.28 \\
\hline Medium & 0.91 & 0.80 to 1.03 & 0.95 & 0.84 to 1.08 & 0.98 & 0.87 to 1.11 \\
\hline Low & 1.17 & 1.03 to 1.32 & 1.10 & 0.97 to 1.24 & 1.14 & 1.01 to 1.28 \\
\hline Very low & 1.19 & 1.02 to 1.39 & 1.16 & 1.00 to 1.35 & 1.18 & 1.01 to 1.36 \\
\hline \multicolumn{7}{|l|}{ Practice size } \\
\hline \multicolumn{7}{|l|}{ Small (reference) } \\
\hline Medium & 0.89 & 0.79 to 1.00 & 0.88 & 0.79 to 0.99 & 0.90 & 0.80 to 1.01 \\
\hline Large & 0.85 & 0.76 to 0.97 & 0.88 & 0.78 to 0.99 & 0.88 & 0.78 to 0.99 \\
\hline \multicolumn{7}{|l|}{ Patient characteristics } \\
\hline \multicolumn{7}{|l|}{ Sex } \\
\hline \multicolumn{7}{|l|}{ Male (reference) } \\
\hline Female & 1.15 & 1.13 to 1.18 & 1.20 & 1.16 to 1.24 & 1.20 & 1.16 to 1.25 \\
\hline \multicolumn{7}{|l|}{ Age category (years) } \\
\hline \multicolumn{7}{|l|}{ 0-19 (reference) } \\
\hline $19-44$ & 2.55 & 2.45 to 2.67 & 2.36 & 2.19 to 2.54 & 2.45 & 2.26 to 2.66 \\
\hline $45-64$ & 2.05 & 1.96 to 2.15 & 2.24 & 2.07 to 2.41 & 2.36 & 2.17 to 2.57 \\
\hline$>65$ & 1.09 & 1.04 to 1.15 & 1.26 & 1.16 to 1.37 & 1.29 & 1.17 to 1.41 \\
\hline \multicolumn{7}{|c|}{ Symptoms of diagnosis (ICPC) } \\
\hline \multicolumn{7}{|c|}{ Other psychological symptoms (reference) } \\
\hline Anxious feelings (1801) & 1.28 & 1.22 to 1.33 & 1.15 & 1.08 to 1.23 & 1.15 & 1.07 to 1.24 \\
\hline Stress (1802) & 1.57 & 1.49 to 1.65 & 1.32 & 1.23 to 1.43 & 1.32 & 1.22 to 1.42 \\
\hline Depressive feelings (1803) & 2.26 & 2.16 to 2.37 & 1.81 & 1.69 to 1.94 & 1.82 & 1.70 to 1.96 \\
\hline Sleeping problems (1806) & 0.19 & 0.18 to 0.20 & 0.19 & 0.17 to 0.21 & 0.18 & 0.16 to 0.21 \\
\hline
\end{tabular}




\begin{tabular}{|c|c|c|c|c|c|c|}
\hline \multirow[b]{3}{*}{ Number of observations in analyses } & \multirow{2}{*}{\multicolumn{2}{|c|}{$\begin{array}{l}\text { (A) Having at least one MHN } \\
\text { consultation per patient per } \\
\text { year (no/yes) } \\
\mathrm{N}=372281 \text { (only patients with } \\
\text { psychological or social } \\
\text { problems) }\end{array}$}} & \multirow{2}{*}{\multicolumn{2}{|c|}{$\begin{array}{l}\text { (B) Number of MHN } \\
\text { consultations per patient per } \\
\text { year }\end{array}$}} & \multirow{2}{*}{\multicolumn{2}{|c|}{$\begin{array}{l}\text { (C) Number of long MHN } \\
\text { consultations per patient per } \\
\text { year } \\
\mathrm{N}=53735 \text { (only patients with at } \\
\text { least one MHN consultation) }\end{array}$}} \\
\hline & & & & & & \\
\hline & $\overline{\text { OR }}$ & $95 \% \mathrm{Cl}$ & $\overline{\mathbf{R R}}$ & $95 \% \mathrm{Cl}$ & $\mathbf{R R}$ & $95 \% \mathrm{Cl}$ \\
\hline Alcohol misuse (1815) & 0.41 & 0.37 to 0.46 & 0.48 & 0.41 to 0.57 & 0.45 & 0.38 to 0.54 \\
\hline Tobacco misuse (1817) & 0.07 & 0.06 to 0.08 & 0.07 & 0.06 to 0.09 & 0.06 & 0.04 to 0.08 \\
\hline Concentration/memory problems (1820) & 0.35 & 0.32 to 0.39 & 0.34 & 0.29 to 0.41 & 0.30 & 0.25 to 0.37 \\
\hline Hyperactive child (1821) & 0.44 & 0.40 to 0.48 & 0.45 & 0.39 to 0.52 & 0.41 & 0.35 to 0.49 \\
\hline Other worries child behaviour (1822) & 0.81 & 0.75 to 0.88 & 0.69 & 0.60 to 0.78 & 0.65 & 0.56 to 0.75 \\
\hline Learning problem (1824) & 0.10 & 0.08 to 0.12 & 0.11 & 0.07 to 0.16 & 0.09 & 0.06 to 0.15 \\
\hline Dementia/Alzheimer (1870) & 0.18 & 0.15 to 0.22 & 0.19 & 0.14 to 0.26 & 0.15 & 0.11 to 0.22 \\
\hline Anxiety disorder (1874) & 1.01 & 0.96 to 1.06 & 1.06 & 0.98 to 1.14 & 1.03 & 0.95 to 1.11 \\
\hline Depression (1876) & 1.21 & 1.15 to 1.26 & 1.30 & 1.22 to 1.39 & 1.28 & 1.20 to 1.37 \\
\hline Neurasthenia (1878) & 2.00 & 1.91 to 2.10 & 1.64 & 1.53 to 1.76 & 1.68 & 1.56 to 1.81 \\
\hline Personality disorder (1880) & 0.97 & 0.89 to 1.06 & 1.12 & 0.99 to 1.27 & 1.06 & 0.93 to 1.21 \\
\hline Other psychological disorders & 0.56 & 0.53 to 0.59 & 0.61 & 0.56 to 0.67 & 0.58 & 0.52 to 0.63 \\
\hline Work problems (2605) & 1.03 & 0.95 to 1.11 & 0.93 & 0.83 to 1.05 & 0.94 & 0.84 to 1.07 \\
\hline Partner/relational problem (2612) & 1.65 & 1.56 to 1.73 & 1.34 & 1.24 to 1.44 & 1.34 & 1.23 to 1.45 \\
\hline III partner (2614) & 0.69 & 0.63 to 0.76 & 0.69 & 0.60 to 0.80 & 0.70 & 0.60 to 0.81 \\
\hline Loss of partner (2615) & 0.59 & 0.54 to 0.65 & 0.67 & 0.58 to 0.77 & 0.67 & 0.58 to 0.77 \\
\hline Child/relational problem (2616) & 1.28 & 1.16 to 1.41 & 1.07 & 0.92 to 1.23 & 1.05 & 0.90 to 1.22 \\
\hline Other social problems & 0.86 & 0.82 to 0.89 & 0.84 & 0.79 to 0.90 & 0.83 & 0.77 to 0.89 \\
\hline \multicolumn{7}{|c|}{$\begin{array}{l}\text { An OR higher than } 1.0 \text { reflects an increased chance of having at least one consultation, compared with the reference group. An RR higher than } 1.0 \text { reflects the chance of having more than one } \\
\text { consultation, compared with the reference group. } \\
\text { Not all ICPC codes in the three diagnosis categories (psychological symptoms, psychological disorders and social problems) are shown in the table. Some diagnoses were less frequent and } \\
\text { combined to limit the number of variables in the model. Other psychological symptoms: all ICPC codes between P01 and P29 not shown in table. Other psychological disorders: all ICPC codes } \\
\text { between P70 and P99 not shown in table. Other social problems: all ICPC codes between Z01 and Z29 not shown in table. 'Other psychological symptoms' was chosen as the reference } \\
\text { category for this variable because of two reasons: first, because the group was relatively large, which was useful for analyses; and second, because patients in this group were represented in } \\
\text { an equal rate at GP and MHN. } \\
\text { GP, general practitioner; ICPC, International Classification of Primary Care; MHN, mental health nurse; RR, risk ratio. }\end{array}$} \\
\hline
\end{tabular}


Figure 2 Mean number of consultations per patient with psychological or social problems seen by Dutch GPs and MHNs between 2010 and 2014 (crude rates). GP, general practitioner; $\mathrm{MHN}$, mental health nurse.

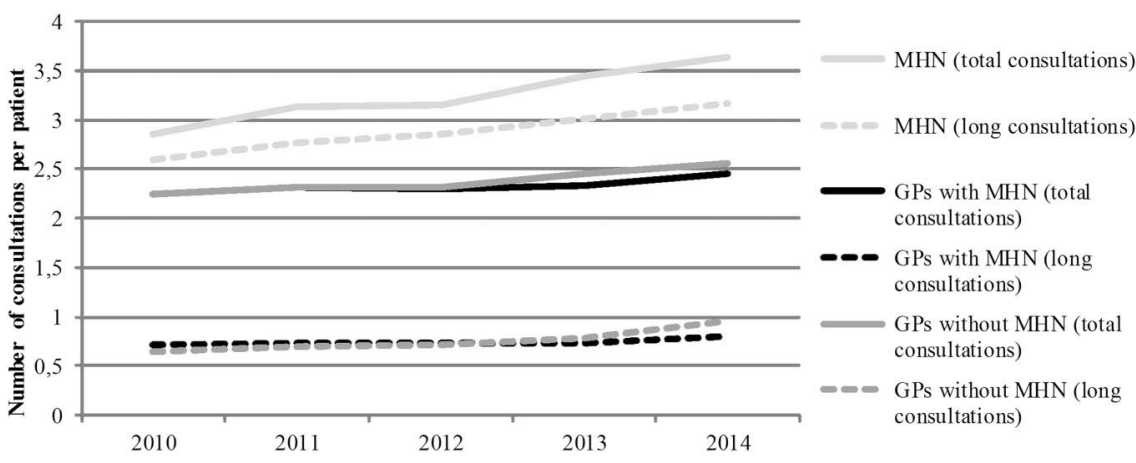

Theoretically, this means that practices we categorised as a practice with MHN could have employed the nurse on the last day of that year. However, since 2011, most practices (around 75\%) employed the MHN since January (see online supplementary file). In 2010, this number was lower $(44 \%)$, but almost $70 \%$ of all practices employed the MHN for at least half a year. We assumed that no or very few practices decided to stop the employment of an MHN during the year. Since many practices employed an MHN for several consecutive years, they were highly likely to be representative of practices with an 'integrated' MHN.

\section{Interpretation of findings and comparison with previous literature}

Since the incidence of mental disorders has been stable for the past decade in the Netherlands, ${ }^{21}$ the observed increase in patients visiting the GP for psychological or social problems may reflect an increasing demand for treatment. This increase seems specific for patients with psychological or social problems, as the number of patients visiting the GP in general or with somatic symptoms seems to be relatively stable over the years. ${ }^{22} 23$

Previous research showed that practice nurses are able to take over care from physicians regarding various

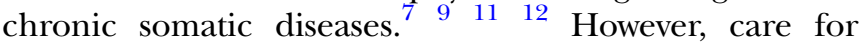
patients with psychological or social problems does not (yet) seem to shift from GPs to MHNs, as Dutch GPs working with an MHN use as many (long) consultations per patient as GPs without an MHN. In fact, GPs who work together with an MHN seem to see more patients with psychological or social problems than GPs without an MHN. GPs who employ an MHN are perhaps more interested in mental health or have improved skills to recognise psychological problems. Often, patients present (vague) somatic problems rather than psychological problems as their primary problem, ${ }^{24}$ and GPs vary in their ability to detect psychological or social problems during consultations. ${ }^{26}$ The presence of an MHN in a general practice may help to make GPs more aware of the importance of recording psychological or social problems during consultations.

The observed increase in number of patients and consultations over time probably reflects an increased capacity in general practice. The MHN is often present for $\sim 1$ day a week, and the number of reimbursed working hours for the MHN was slowly increased over the past few years. This increased capacity may influence GPs' behaviour; the possibility to offer short psychological treatment within their own practice may increase their likeliness to record psychological or social problems. In this way, MHNs may contribute to improved accessibility

Our finding that MHNs probably do not reduce GP workload, but provide additional support, is in line with a previous study on collaborative care, involving a depression care manager in primary care and consultation of mental health specialists. ${ }^{27}$ Within that study, no differences were found in resource use, including GP care, between patients in usual care and patients in collaborative care. However, a Cochrane review on counselling in primary care suggested that although counselling in primary care does not seem to reduce (total) healthcare costs, it may reduce some types of healthcare usage. ${ }^{13}$ Another review on psychiatric consultation in primary care also concluded that it may reduce usage of healthcare services. ${ }^{28}$ Moreover, a Cochrane review on the effects of mental health workers in primary care concluded that their presence might decrease consultation rates, prescriptions of psychotropic drugs and referrals to specialists. However, effects were modest and results were not consistent among all included studies. ${ }^{29}$ An alternative explanation for the similar workload of GPs with and without an MHN found in this study might be that 1-day support a week is not enough to significantly change care. Indeed, the ministry of health has proposed to increase the number of MHN working hours per practice to 40 per week. Future studies will show what the effect of these extra hours is on the recognition and referral of patients with psychological or social problems.

This study showed that MHNs treat certain GP patients more often: females, adults and patients with common psychological symptoms such as depressive or anxious feelings. This finding might be related to the fact that most MHNs are educated in general adult care, and not for the treatment of specific subgroups, such as children. Besides, some patient groups, such as the elderly, might not want to be treated by an MHN themselves. Lastly, the MHN's general knowledge may not be of mental healthcare. 
sufficient for complete diagnosis or treatment of all patients, even when they do not have a DSM-IV disorder and thus officially should be treated within general practice. This seems especially plausible for specific subgroups whose treatment requires expertise, such as children. Large group practices with multiple GPs might be able to collaborate with several MHNs with varying expertise, for example, in youth or elderly mental healthcare.

It is not clear to what extent MHNs recognise and treat patients with comorbid problems. Presumably, the presence of an MHN in a general practice increases the chance that comorbid psychological problems are detected. Patients with primarily somatic problems may sometimes receive counselling or support from the MHN to learn how to manage a somatic disease or comorbid depressive or anxious feelings. Previous research in the UK suggests that the primary care setting is appropriate for the support of people with chronic psychological diseases $^{3031}$ or comorbid somatic diseases. ${ }^{32}$

Although in some other countries nurses similar to Dutch MHNs are working in general practices, it is not clear to what extent our results are generalisable. Factors influencing a possible shift of care from GPs to MHNs may vary strongly between countries, such as task roles, workload, (financial) regulations and patient expectations. This study also cannot conclude as to the effectiveness of treatment by MHNs. Previous research showed that although psychosocial treatment in primary care may be effective, ${ }^{14-16}$ it might be less effective than treatment in a specialist setting, possibly because of the shorter treatment duration. ${ }^{33}$ It is highly likely that some of the patients visiting the MHN still need treatment in specialised care, especially when diagnostics are the main reason for MHN consultation. It is plausible that the presence of MHNs in general practice influences the number of patients treated in other settings of mental healthcare, such as specialised care. The increase in patients in general practice will possibly be accompanied by a decrease in patients in specialised care, as the early treatment of patients might prevent them from needing further treatment. On the other hand, it is also possible that as more patients are detected and referred by GPs and MHNs, more will be treated by specialists. To study the effects of the MHN on the patient numbers in other settings of mental healthcare, future research is needed which combines data from NIVEL-PCD with national data from specialised care.

\section{CONCLUSIONS}

MHNs and GPs in the Netherlands treat increasing numbers of patients for psychological and social problems. MHNs do not seem to replace GP care, but probably deliver additional consultations to patients. GPs with an MHN see slightly more patients with psychological or social problems than GPs without an MHN. Future research should study to what extent early treatment of patients within the general practice prevents them from needing specialised care.

Acknowledgements The authors would like to thank Peter Spreeuwenberg for his contribution to the statistical analyses.

Contributors TM, DPdB, DHdB and PFV designed the study. TM analysed the data and wrote the first draft of the manuscript. PS contributed to the statistical analyses. All authors contributed to and approved the final manuscript.

Funding This study was carried out with a grant of the Dutch Ministry of Health, Welfare and Sport.

\section{Competing interests None declared.}

Ethics approval Dutch law allows the use of electronic health records for research purposes under certain conditions. According to this legislation, neither obtaining informed consent from patients nor approval by a medical ethics committee is obligatory for this type of observational studies containing no directly identifiable data. ${ }^{34}$ This study has been approved by the applicable governance bodies of the NIVEL Primary Care Database under no. NZR-003.15.003.

Provenance and peer review Not commissioned; externally peer reviewed. Data sharing statement No additional data are available.

Open Access This is an Open Access article distributed in accordance with the Creative Commons Attribution Non Commercial (CC BY-NC 4.0) license, which permits others to distribute, remix, adapt, build upon this work noncommercially, and license their derivative works on different terms, provided the original work is properly cited and the use is non-commercial. See: http:// creativecommons.org/licenses/by-nc/4.0/

\section{REFERENCES}

1. World Health Organization (WHO). Integrating mental health into primary care: a global perspective. WHO Publishing, 2008. http:// www.who.int/mental_health/resources/mentalhealth_PHC_2008. pdf (accessed 22 Dec 2015).

2. Organisation for Economic Co-operation and Development (OECD). Focus on health: making mental health count. OECD Publishing, 2014 (cited 22 December 2015). http://www.oecd.org/ els/health-systems/Focus-on-Health-Making-Mental-HealthCount.pdf

3. WHO. Mental health action plan 2013-2020. WHO Publishing, 2013 (cited 22 December 2015). http://www.who.int/mental_health/ publications/action_plan/en/

4. Verhaak PF, van de Lisdonk EH, Bor JH, et al. GPs' referral to mental health care during the past 25 years. Br J Gen Pract 2000;50:307-8.

5. Verhaak PF, van Dijk CE, Nuijen J, et al. Mental health care as delivered by Dutch general practitioners between 2004 and 2008. Scand J Prim Health Care 2010;30:156-62.

6. American Psychiatric Association (APA). Diagnostic and statistical manual of mental disorders (4th ed., text rev.). Washington DC: American Psychiatric Association, 2000.

7. Laurant M, Reeves D, Hermens R, et al. Substitution of doctors by nurses in primary care. Cochrane Database Syst Rev 2005;(2): CD001271.

8. Dierick-van Daele AT, Spreeuwenberg C, Derckx EW, et al. The value of nurse practitioners in Dutch general practices. Qual Prim Care 2010;18:231-41.

9. Martinez-Gonzalez NA, Djalali S, Tandjung R, et al. Substitution of physicians by nurses in primary care: a systematic review and meta-analysis. BMC Health Serv Res 2014;14:214.

10. Dierick-van Daele AT, Steuten LM, Metsemakers JF, et al. Economic evaluation of nurse practitioners versus GPs in treating common conditions. Br J Gen Pract 2010;60:e28-35.

11. Freund $T$, Everett $C$, Griffiths $P$, et al. Skill mix, roles and remuneration in the primary care workforce: who are the healthcare professionals in the primary care teams across the world? Int $J$ Nurs Stud 2015;52:727-43.

12. Van Dijk CE, Verheij RA, Hansen J, et al. Primary care nurses: effects on secondary care referrals for diabetes. BMC Health Serv Res 2010;10:230. 
13. Bower P, Knowles S, Coventry PA, et al. Counselling for mental health and psychosocial problems in primary care. Cochrane Database Syst Rev 2011;(9):CD001025.

14. Huibers MJ, Beurskens AJ, Bleijenberg G, et al. Psychosocial interventions by general practitioners. Cochrane Database Syst Rev 2007;(3):CD003494.

15. Kendrick T, Simons L, Mynors-Wallis L, et al. Cost-effectiveness of referral for generic care or problem-solving treatment from community mental health nurses, compared with usual general practitioner care for common mental disorders: randomised controlled trial. Br J Psychiatry 2006;189:50-9.

16. Van Orden M, Hoffman T, Haffmans J, et al. Collaborative mental health care versus care as usual in a primary care setting: a randomized controlled trial. Psychiatr Serv 2009; 60:74-9.

17. NIVEL Primary Care Database (NIVEL Zorgregistraties eerste lijn). Netherlands institute for health services research. 2015. http://www. nivel.nl/en/dossier/nivel-primary-care-database (accessed 22 Dec 2015).

18. Van Hassel DTP, Kasteleijn A, Kenens RJ. Cijfers uit de registratie van huisartsen: peiling 2013 [Numbers from the registrations of general practitioners: poll 2013]. Utrecht: NIVEL, 2014.

19. Merlo J, Chaix B, Ohlsson $\mathrm{H}$, et al. A brief conceptual tutorial of multilevel analysis in social epidemiology: using measures of clustering in multilevel logistic regression to investigate contextual phenomena. J Epidemiol Community Health 2006;60:

290-7.

20. Landelijke Huisartsen Vereniging (LHV). Tweede peiling GGZ [Second poll mental health care]. https://www.lhv.nl/actueel/nieuws/ huisarts-vangnet-voor-patienten-met-psychische-problemen (accessed 22 Dec 2015)

21. De Graaf R, Ten Have M, Van Gool S, et al. Prevalentie van psychische aandoeningen en trends van 1996 tot 2009; resultaten van NEMESIS-2 [Prevalence of psychological diseases and trends between 1996 and 2009; results of NEMESIS-2]. Tijdschr Psychiatr 2012;54:27-38.

22. Prins MA, Davids R, Verheij RA. Huisarts-Omvang zorggebruik. NIVEL Zorgregistraties eerste lijn [General practitioner-extent of health care use. NIVEL Primary Care Database]. http://www.nivel.nl/ node/3166 (accessed 18 Feb 2016).
23. Wildeboer RM, van der Hoek L, Verhaak PFM. Use of GP services 5 years after an episode of mental illness: case-control study using electronic records. Br J Gen Pract 2016;66:e347-53.

24. Cape J. How general practice patients with emotional problems presenting with somatic or psychological symptoms explain their improvement. Br J Gen Pract 2001;51:724-9.

25. Sayal K, Taylor E. Detection of child mental health disorders by general practitioners. Br J Gen Pract 2004;54:348-52.

26. Zantinge EM, Verhaak PF, de Bakker DH, et al. The workload of general practitioners does not affect their awareness of patients' psychological problems. Patient Educ Couns 2007;67:93-9.

27. Green C, Richards DA, Hill JJ, et al. Cost-effectiveness of collaborative care for depression in UK primary care: economic evaluation of a randomised controlled trial (CADET). PLOS ONE 2014:9:e104225.

28. van der Feltz-Cornelis CM, Van Os TW, Van Marwijk HW, et al. Effect of psychiatric consultation models in primary care. A systematic review and meta-analysis of randomized clinical trials. $J$ Psychosom Res 2010;68:521-33.

29. Harkness EF, Bower PJ. On-site mental health workers delivering psychological therapy and psychosocial interventions to patients in primary care: effects on the professional practice of primary care providers. Cochrane Database Syst Rev 2009;(1):CD000532.

30. Miller CJ, Grogan-Kaylor A, Perron BE, et al. Collaborative chronic care models for mental health conditions: cumulative meta-analysis and metaregression to guide future research and implementation. Med Care 2013;51:922-30.

31. Richards DA, Hill JJ, Gask L, et al. Clinical effectiveness of collaborative care for depression in UK primary care (CADET): cluster randomised controlled trial. BMJ 2013;347:f4913.

32. Coventry $\mathrm{P}$, Lovell $\mathrm{K}$, Dickens $\mathrm{C}$, et al. Integrated primary care for patients with mental and physical multimorbidity: cluster randomised controlled trial of collaborative care for patients with depression comorbid with diabetes or cardiovascular disease. BMJ 2015;350: h638.

33. Cape J, Whittington C, Buszewicz M, et al. Brief psychological therapies for anxiety and depression in primary care: meta-analysis and meta-regression. BMC Med 2010;8:38.

34. Dutch Civil Law, Article 7:458. http://www.dutchcivillaw.com/ civilcodebook077.htm (accessed 22 Dec 2015). 